\title{
Diophantine approximation for conformal measures of one-dimensional iterated function systems
}

\author{
Mariusz Urbański
}

\begin{abstract}
Recall that a Borel measure $\mu$ on $\mathbb{R}$ is said to be extremal if $\mu$-almost every number in $\mathbb{R}$ is not very well approximable. In this paper, we investigate extremality (implied by the exponentially fast decay (efd) property) of conformal measures induced by regular infinite conformal iterated function systems. We then give particular attention to the class of such systems generated by the continued fractions algorithm with restricted entries. It is proved that if the index set of entries has bounded gaps, then the corresponding conformal measure satisfies the efd property and is extremal. Also a class of examples of index sets with unbounded gaps is provided for which the corresponding conformal measure also satisfies the efd property and is extremal.
\end{abstract}

\section{Introduction}

A point $x \in \mathbb{R}$ is said to be very well approximable if there exist $\delta>0$ and infinitely many integers $p, q \in \mathbb{Z}, q \geqslant 1$, such that

$$
|q x-p| \leqslant q^{-(1+\delta)} .
$$

It is a classical result that the set of all very well approximable numbers has the Lebesgue measure zero but the Hausdorff dimension equal to one. Thus the natural question arises about other measures. To be more precise, a Borel measure $\mu$ on $\mathbb{R}$ is said to be extremal if $\mu$-almost every number in $\mathbb{R}$ is not very well approximable. Barak Weiss in [Wei01] provided a nice sufficient condition for a Borel probability measure on $\mathbb{R}$ to be extremal. We will use this result heavily. We would like to add that Weiss's result has its multidimensional counterparts (see [KM98], [KLW04], [PV] and [Urb] for more examples).

In this paper, we investigate extremality (implied by the exponentially fast decay (efd) property) of conformal measures induced by regular infinite conformal iterated function systems. We recall that the iteration of infinitely many conformal maps naturally arises in several contexts. For example, one is naturally led to consider infinite systems when one is dealing with parabolic systems, i.e. finite systems of conformal maps which have some cusps (see [MU03a] and the references therein). Another natural class of examples, investigated in detail in our article, arises from continued fractions with restricted entries.

In the present paper our ultimate goal is to explore extremality of conformal measures naturally associated to regular continued fraction iterated function systems with restricted entries. Our starting point is the above-mentioned result of Barak Weiss (see Theorem 3.2; cf. [Wei01]) saying that the efd property introduced in [Vee99] by Veech, is sufficient for extremality in the one-dimensional case (the support of the reference measure is contained in $\mathbb{R}$ ). In our paper, the extremality of

Received 17 December 2003, accepted in final form 7 August 2004, published online 21 June 2005.

2000 Mathematics Subject Classification 11J70 (primary), 37D35, 37E99 (secondary).

Keywords: not very well approximable numbers, exponentially fast decaying measures, extremal measures, continued fractions, conformal iterated function systems.

This journal is (C) Foundation Compositio Mathematica 2005. 


\section{URBAŃSKI}

conformal measures we are dealing with is always proven via the efd property. In a forthcoming paper, Kleinbock and Weiss derive some additional diophantine consequences of the efd property.

Our paper is organized as follows. In $\S 2$ (Preliminaries) we collect some basic properties of conformal infinite iterated function systems. In $\S 3$ (General sufficient conditions) we establish the efd property of several classes of measures and, as the main result of the section, we prove Theorem 3.7, a sufficient condition for the efd property to hold, which is heavily used in further sections. In $\S 4$ we consider the maps $\phi_{n}:[0,1] \rightarrow[0,1]$, for $n \in \mathbb{N}$, given by the formula

$$
\phi_{n}(x)=\frac{1}{x+n},
$$

and an arbitrary subset $I$ of positive integers $\mathbb{N}$. We investigate the corresponding iterated function system $S_{I}=\left\{\phi_{i}\right\}_{i \in I}$, which is called a continued fraction iterated function system. Its limit set $J_{I}$ consists of all those $x \in(0,1)$ for which each partial denominator $x_{i}, i \geqslant 1$, in the continued fraction expansion

$$
x=\frac{1}{x_{1}+\frac{1}{x_{2}+\frac{1}{x_{3}+\frac{1}{\ddots}}}}
$$

is in $I$. In Theorem 5.1 an effective sufficient condition for the conformal measure of the system $S_{I}$ to satisfy the efd property (and, as a consequence, to be extremal) is provided. This condition is expressed in terms of the arithmetic properties of the infinite set $I$ of positive integers. This continues our theme from [MU99], [Urb01a], [MU03b] that many geometric measure theoretic properties of these systems are reflected in the arithmetic properties of the index set $I$. The last section, $\S 5$, is occupied by the proof of the following two facts: first, that each index set $I$ with bounded gaps gives rise to the conformal measure (which, up to a multiplicative constant, is equal to the packing measure on $J_{I}$ ) which satisfies the efd property and, in consequence, is extremal; secondly, that there exist infinite regular systems with unbounded gaps whose conformal measures satisfy the efd property and are extremal.

\section{Preliminaries}

First, let us describe the setting of conformal (infinite) iterated function systems introduced in [MU96]. Let $I$ be a countable index set or alphabet with at least two elements and let $S=\left\{\phi_{i}\right.$ : $X \rightarrow X\}_{i \in I}$ be a collection of injective contractions from a compact metric space $X$ into $X$ for which there exists $0<s<1$ such that $\rho\left(\phi_{i}(x), \phi_{i}(y)\right) \leqslant s \rho(x, y)$, for every $i \in I$ and for every pair of points $x, y \in X$. Thus, the system $S$ is uniformly contractive. Any such collection $S$ of contractions is called an iterated function system. We define the limit set $J$ of this system as the image of the coding space under a coding map as follows. Let $I^{n}$ denote the space of words of length $n, I^{\infty}$ the space of infinite sequences of symbols in $I, I^{*}=\bigcup_{n \geqslant 1} I^{n}$ and, for $\omega \in I^{n}, n \geqslant 1$, let $\phi_{\omega}=\phi_{\omega_{1}} \circ \phi_{\omega_{2}} \circ \cdots \circ \phi_{\omega_{n}}$. If $\omega \in I^{*} \cup I^{\infty}$ and $n \geqslant 1$ does not exceed the length of $\omega$, we denote by $\left.\omega\right|_{n}$ the word $\omega_{1} \omega_{2} \ldots \omega_{n}$. Since, given $\omega \in I^{\infty}$, the diameters of the compact sets $\phi_{\left.\omega\right|_{n}}(X), n \geqslant 1$, converge to zero and since they form a descending family, the set

$$
\bigcap_{n=0}^{\infty} \phi_{\left.\omega\right|_{n}}(X)
$$

is a singleton and therefore, denoting its only element by $\pi(\omega)$, it defines the coding map $\pi: I^{\infty} \rightarrow X$. The main object of our interest will be the limit set

$$
J=J_{S}=\pi\left(I^{\infty}\right)=\bigcup_{\omega \in I^{\infty}} \bigcap_{n=1}^{\infty} \phi_{\omega \mid n}(X) .
$$




\section{Diophantine APPROXimation FOR CONFORMAL MEASURES}

An iterated function system $S=\left\{\phi_{i}: X \rightarrow X\right\}_{i \in I}$ is said to satisfy the Open Set Condition (OSC) if there exists a nonempty open set $U \subset X$ (in the topology of $\mathrm{X}$ ) such that $\phi_{i}(U) \subset U$ for every $i \in I$ and $\phi_{i}(U) \cap \phi_{j}(U)=\emptyset$ for every pair $i, j \in I, i \neq j$. (We do not exclude the possibility that $\overline{\phi_{i}(U)} \cap \overline{\phi_{j}(U)} \neq \emptyset$.) A system satisfying the OSC is said to satisfy the Strong Open Set Condition (SOSC) if $J_{S} \cap U \neq \emptyset$ and it is said to satisfy the Super-Strong Open Set Condition (SSOSC) if $\overline{J_{S}} \subset U$.

An iterated function system $S$ satisfying the OSC is said to be conformal if $X \subset \mathbb{R}^{d}$ for some $d \geqslant 1$ and the following conditions are satisfied.

(1a) $U=\operatorname{Int}_{\mathbb{R}^{d}}(X)$.

(1b) There exists an open connected set $V$ such that $X \subset V \subset \mathbb{R}^{d}$ and such that all maps $\phi_{i}$, $i \in I$, extend to $C^{1}$ conformal diffeomorphisms of $V$ into $V$. (Note that for $d=1$ this just means that all the maps $\phi_{i}, i \in I$, are $C^{1}$ monotone diffeomorphisms, for $d=2$ the words $C^{1}$ conformal mean holomorphic or antiholomorphic, and for $d>2$ the maps $\phi_{i}, i \in I$, are Möbius transformations. The proof of the last statement can be found, for example, in [BP92], where it is called Liouville's theorem.)

(1c) There exist $\gamma, l>0$ such that for every $x \in X \subset \mathbb{R}^{d}$ there exists an open cone $\operatorname{Con}(x, \gamma, l) \subset$ $\operatorname{Int}(X)$ with vertex $x$, central angle of Lebesgue measure $\gamma$, and altitude $l$.

Notice that if either $d \geqslant 2$ (see [MU03a]; cf. [Urb01b]) or if the system $S$ is finite with $\phi_{i} \in C^{1+\varepsilon}$ for all $i \in I$, then the following two conditions are satisfied, the second being a strengthening of the first.

(1d) Bounded Distortion Property (BDP). There exists $K \geqslant 1$ such that

$$
\left|\phi_{\omega}^{\prime}(y)\right| \leqslant K\left|\phi_{\omega}^{\prime}(x)\right|
$$

for every $\omega \in I^{*}$ and every pair of points $x, y \in V$, where $\left|\phi_{\omega}^{\prime}(x)\right|$ means the norm of the derivative.

(1e) There exists a function $K:[0, \infty) \rightarrow \mathbb{R}$ such that $\lim _{r \searrow_{0}} K(r)=1$ and

$$
\left|\phi_{\omega}^{\prime}(y)\right| \leqslant K(\|x-y\|)\left|\phi_{\omega}^{\prime}(x)\right|
$$

for every $\omega \in I^{*}$ and every pair of points $x, y \in V$.

In the case when $d=1$ and $I$ is infinite, the conditions (1d) and (1e) form extra assumptions that are attached to the definition of a conformal iterated function system.

In the following we will use the terminology and results taken from $\S 1$ (Preliminaries) of [MU03b]. Here we provide only the definition of a conformal measure, the main object dealt with in this paper. If $S$ is a conformal iterated function system, a Borel probability measure $m$ is said to be $t$-conformal provided $m\left(J_{S}\right)=1$ and, for every Borel set $A \subset X$ and every $i \in I$,

$$
m\left(\phi_{i}(A)\right)=\int_{A}\left|\phi_{i}^{\prime}\right|^{t} d m
$$

and

$$
m\left(\phi_{i}(X) \cap \phi_{j}(X)\right)=0
$$

for every pair $i, j \in I, i \neq j$.

If a $t$-conformal measure exists, then $t=\operatorname{HD}(J)$, the Hausdorff dimension of the limit set $J$, denoted in the following by $h$. If a conformal measure exists, it is unique. 


\section{URBAŃSKI}

\section{General sufficient conditions}

We start with the following simple but useful result.

Proposition 3.1. Let $(X, \rho)$ be a metric space and let $\mu$ be a Borel probability measure on $X$. Then the following two conditions are equivalent.

$$
\begin{gathered}
\exists(\alpha \in(0,1)) \exists(\beta>1) \exists(\xi>0) \forall(x \in X) \forall(r \leqslant \xi) \\
\mu(B(x, r)) \leqslant \alpha \mu(B(x, \beta r)) .
\end{gathered}
$$

(b) There exists a Borel set $Y \subset X$ with $\mu(Y)=1$ such that condition (a) is satisfied with $X$ replaced by $Y$.

Proof. The implication $(\mathrm{a}) \Rightarrow(\mathrm{b})$ is obvious. In order to prove the opposite implication fix $x \in X$ and $r \leqslant \xi / 2$. If $B(x, r) \cap Y=\emptyset$, then $\mu(B(x, r))=0$, and we are done. Otherwise, fix $y \in B(x, r) \cap Y$. Then

$$
\mu(B(x, r)) \leqslant \mu(B(y, 2 r)) \leqslant \alpha \mu(B(y, \beta 2 r)) \leqslant \alpha \mu(B(x, 2 \beta r+r))=\alpha \mu(B(x,(2 \beta+1) r)),
$$

and we are done.

Any Borel probability measure satisfying condition (a) or, equivalently, condition (b) of Proposition 3.1, is said to satisfy the efd property. The significance of this property, although interesting itself, results from the following fact, essentially proven in [Wei01] and crucial for our approach.

Theorem 3.2 (Weiss). Every Borel probability measure on $\mathbb{R}$ satisfying the efd property is extremal.

A Borel probability measure $\mu$ is said to be geometric if there exist $\xi>0, C>0$, and $h>0$ such that

$$
C^{-1} r^{h} \leqslant \mu(B(x, r)) \leqslant C r^{h}
$$

for all $x \in \operatorname{supp}(\mu)$ and all $r \leqslant \xi$. Our next simple result is the following.

Proposition 3.3. Every geometric measure satisfies the efd property.

Proof. Fix $\beta>1$ to be specified later and consider an arbitrary point $x \in \operatorname{supp}(\mu)$ and $r \leqslant \xi / \beta$. Then

$$
\mu(B(x, r)) \leqslant C r^{h}=C^{2} \beta^{-h}\left(C^{-1}(\beta r)^{h}\right) \leqslant C^{2} \beta^{-h} \mu(B(x, \beta r)),
$$

and it suffices to take $\beta>1$ so large that $C^{2} \beta^{-h}<1$. We are therefore done since the condition (b) of Proposition 3.1 is satisfied with $Y=\operatorname{supp}(\mu)$.

As an immediate consequence of this proposition, Lemma 3.14 from [MU96] and Theorem 3.2, we get the following theorem.

Theorem 3.4. If $S=\left\{\phi_{i}: X \rightarrow X\right\}_{i \in I}$ is a finite conformal iterated function system and $h:=$ $\mathrm{HD}\left(J_{S}\right)$, then all the Hausdorff $\left.\mathrm{H}^{s}\right|_{J_{S}}$ and packing $\left.\mathrm{P}^{s}\right|_{J_{S}}$ measures and the h-conformal measure $m$ on $J_{S}$ satisfy the efd property. If, in addition, $X \subset \mathbb{R}$, then all these measures are extremal.

As an immediate consequence of Proposition 3.3 and a well-known theorem from [PU], we get the following theorem.

Theorem 3.5. If $f: X \rightarrow X$ is a mixing expanding repeller and $h:=\operatorname{HD}(X)$, then the $h$-dimensional Hausdorff measure on $X$ and the $h$-dimensional packing measure on $X$ satisfy the efd property. If, in addition, $X \subset \mathbb{R}$, then all these measures are extremal. 


\section{DiOPHANTINE APPROXIMATION FOR CONFORMAL MEASURES}

We shall now prove, in the context of iterated function systems, a sufficient condition for a conformal measure to satisfy the efd property. This condition will be the starting point of all of our more specific results establishing the efd property of conformal measures for some conformal iterated function systems.

Theorem 3.6. Suppose that the system $S=\left\{\phi_{i}\right\}_{i \in I}$ is regular, and denote by $m$ the corresponding $h$-conformal measure. Suppose also that there exists an integer $q \geqslant 0$ and $\kappa \in(0, \min \{1$, $\operatorname{dist}(X, \partial V)\})$ such that the following three conditions are satisfied.

(a) There exist three real constants $\gamma \geqslant 1, \beta>1, \alpha \in(0,1)$, and a finite set $F \subset I$ such that, for all $i \in I \backslash F$, all $x \in \phi_{i}(J)$, and all $r \in\left[\gamma\left\|\phi_{i}^{\prime}\right\|, \kappa\right]$,

$$
m(B(x, r)) \leqslant \alpha \mu(B(x, \beta r)) .
$$

(b) For all $\omega \in I^{*}$ with $|\omega| \leqslant q$, all $x \in J$, and all $r \in(0, \kappa)$,

$$
m\left(\phi_{\omega}(B(x, r))\right) \leqslant \alpha^{-1 / 3}\left|\phi_{\omega}^{\prime}(x)\right|^{h} m(B(x, r)) .
$$

(c) For all $\omega \in I^{q}$ and all $x \in J$,

$$
\phi_{\omega}(B(x, \kappa)) \subset X .
$$

Then the $h$-conformal measure $m$ satisfies the efd property.

Proof. Let

$$
G=F \cup\left\{i \in I: \gamma\left\|\phi_{i}^{\prime}\right\|>\kappa\right\} .
$$

Since $\lim _{i \in I}\left\|\phi_{i}^{\prime}\right\|=0$, the set $G$ is finite. Now take an arbitrary $\rho>0, i \in I \backslash G, x \in \phi_{i}(J)$, and $r \in\left(\rho\left\|\phi_{i}^{\prime}\right\|, \kappa\right)$. If $r \geqslant \gamma\left\|\phi_{i}^{\prime}\right\|$, then it follows from condition (a) that $m(B(x, r)) \leqslant \alpha \mu(B(x, \beta r))$. If $r<\gamma\left\|\phi_{i}^{\prime}\right\|$, then $\rho<\gamma$, and it follows from condition (a) that

$$
\begin{aligned}
m(B(x, r)) & \leqslant m\left(B\left(x, \gamma\left\|\phi_{i}^{\prime}\right\|\right)\right) \leqslant \alpha m\left(B\left(x, \beta \gamma\left\|\phi_{i}^{\prime}\right\|\right)\right) \leqslant \alpha m\left(B\left(x, \beta \frac{\gamma}{\rho} \rho\left\|\phi_{i}^{\prime}\right\|\right)\right) \\
& \leqslant \alpha m\left(B\left(x, \beta \frac{\gamma}{\rho} r\right)\right) .
\end{aligned}
$$

In any case

$$
m(B(x, r)) \leqslant \alpha m(B(x, \beta \max \{1, \gamma / \rho\} r)) .
$$

Put $\eta=\min \left\{\left\|\phi_{i}^{\prime}\right\|: i \in G\right\}$. Since the measure $m$ is positive on non-empty open subsets of $J$, we have $P:=\inf \{m(B(x, \gamma \beta \eta)): x \in J\}>0$. Since the measure $m$ has no atoms, there exists $\zeta \in(0, \kappa)$ so small that

$$
m(B(x, \zeta)) \leqslant \alpha P
$$

for all $x \in J$. In view of condition (1e) we may assume $\zeta \in(0, \kappa)$ to be so small that

$$
\alpha^{\frac{1}{6 h}} \leqslant \frac{\left|\phi_{\omega}^{\prime}(y)\right|}{\left|\phi_{\omega}^{\prime}(x)\right|} \leqslant \alpha^{-\frac{1}{6 h}}
$$

for all $\omega \in I^{*}$, all $x \in J$, and all $y \in B(x, \zeta)$. Similarly as above, $Q:=\inf \{m(B(x, \zeta)): x \in J\}>0$ and there exists $\theta \in(0, \zeta / K)$ so small that

$$
m(B(x, K \theta)) \leqslant \alpha Q
$$

for all $x \in J$. Now fix $x \in J$ and $r \in(0, \theta)$. Write $x=\pi(\omega)$, where $\omega \in I^{\infty}$, and let $n \geqslant 0$ be the least integer such that $\left\|\phi_{\left.\omega\right|_{n}}^{\prime}\right\| \leqslant \theta^{-1} r$. Then $n \geqslant 1$ and $\left\|\phi_{\left.\omega\right|_{n-1}}^{\prime}\right\|>\theta^{-1} r$. Consequently,

$$
K r\left\|\phi_{\left.\omega\right|_{n-1}}^{\prime}\right\|^{-1} \geqslant r\left\|\phi_{\left.\omega\right|_{n}}^{\prime}\right\|^{-1}\left\|\phi_{\omega_{n}}^{\prime}\right\| \geqslant \theta\left\|\phi_{\omega_{n}}^{\prime}\right\|
$$

and

$$
K r\left\|\phi_{\left.\omega\right|_{n-1}}^{\prime}\right\|^{-1}<K \theta<\zeta<\kappa<\operatorname{dist}(X, \partial V) .
$$




\section{URBAŃSKI}

This inequality along with formula (1.4) from [MU03b] imply that

$$
B(x, r) \subset \phi_{\left.\omega\right|_{n-1}}\left(B\left(\pi\left(\sigma^{n-1}(\omega)\right), K r\left\|\phi_{\left.\omega\right|_{n-1}}^{\prime}\right\|^{-1}\right)\right),
$$

and, if $n-1 \leqslant q$, it then follows from condition (b) that

$$
m(B(x, r)) \leqslant \alpha^{-1 / 3}\left|\phi_{\left.\omega\right|_{n-1}}^{\prime}\left(\pi\left(\sigma^{n-1}(\omega)\right)\right)\right|^{h} m\left(B\left(\pi\left(\sigma^{n-1}(\omega)\right), K r\left\|\phi_{\left.\omega\right|_{n-1}}^{\prime}\right\|^{-1}\right)\right) .
$$

If $n-1>q$, then write $\left.\omega\right|_{n-1}=\tau \eta$, where $|\eta|=q$. It then follows from (3.6), condition (c), (3.3), and condition (b) that

$$
\begin{aligned}
m(B(x, r)) & \leqslant m\left(\phi_{\tau}\left(\phi_{\eta}\left(B\left(\pi\left(\sigma^{n-1}(\omega)\right), K r\left\|\phi_{\left.\omega\right|_{n-1}}^{\prime}\right\|^{-1}\right)\right)\right)\right) \\
& \leqslant \alpha^{-1 / 6}\left|\phi_{\tau}^{\prime}\left(\phi_{\eta}\left(\pi\left(\sigma^{n-1}(\omega)\right)\right)\right)\right|^{h} m\left(\phi_{\eta}\left(B\left(\pi\left(\sigma^{n-1}(\omega)\right), K r\left\|\phi_{\left.\omega\right|_{n-1}}^{\prime}\right\|^{-1}\right)\right)\right) \\
& \leqslant \alpha^{-1 / 6}\left|\phi_{\tau}^{\prime}\left(\phi_{\eta}\left(\pi\left(\sigma^{n-1}(\omega)\right)\right)\right)\right|^{h} \alpha^{-1 / 3}\left|\phi_{\eta}^{\prime}\left(\pi\left(\sigma^{n-1}(\omega)\right)\right)\right|^{h} m\left(B\left(\pi\left(\sigma^{n-1}(\omega)\right), K r\left\|\phi_{\left.\omega\right|_{n-1}}^{\prime}\right\|^{-1}\right)\right. \\
& =\alpha^{-1 / 2}\left|\phi_{\left.\omega\right|_{n-1}}^{\prime}\left(\pi\left(\sigma^{n-1}(\omega)\right)\right)\right|^{h} m\left(B\left(\pi\left(\sigma^{n-1}(\omega)\right), K r\left\|\phi_{\left.\omega\right|_{n-1}}^{\prime}\right\|^{-1}\right) .\right.
\end{aligned}
$$

It now follows from (3.7), (3.8), (3.6), and (3.4) that

$$
\begin{aligned}
m(B(x, r)) & \leqslant \alpha^{-1 / 2}\left|\phi_{\left.\omega\right|_{n-1}}^{\prime}\left(\pi\left(\sigma^{n-1}(\omega)\right)\right)\right|^{h} \alpha m\left(B\left(\pi\left(\sigma^{n-1}(\omega)\right), \zeta\right)\right) \\
& =\alpha^{1 / 2}\left|\phi_{\left.\omega\right|_{n-1}}^{\prime}\left(\pi\left(\sigma^{n-1}(\omega)\right)\right)\right|^{h} 2 \phi_{n} m\left(B\left(\pi\left(\sigma^{n-1}(\omega)\right), \zeta\right)\right) .
\end{aligned}
$$

Now consider the case when $\gamma \theta^{-1} \beta K r\left\|\phi_{\left.\omega\right|_{n-1}}^{\prime}\right\|^{-1} \geqslant \zeta$. Then, in view of the Mean Value Inequality and (3.3), we get that

$$
m\left(B\left(x, \gamma \theta^{-1} \beta K r\right)\right) \geqslant \alpha^{1 / 6}\left|\phi_{\left.\omega\right|_{n-1}}^{\prime}\left(\pi\left(\sigma^{n-1}(\omega)\right)\right)\right|^{h} m\left(B\left(\pi\left(\sigma^{n-1}(\omega)\right), \zeta\right)\right) .
$$

Combining these last two inequalities, we obtain

$$
m(B(x, r)) \leqslant \alpha^{1 / 3} m\left(B\left(x, \gamma \theta^{-1} \beta K r\right)\right) .
$$

So, suppose that

Then in view of $(3.3)$,

$$
\gamma \theta^{-1} \beta K r\left\|\phi_{\left.\omega\right|_{n-1}}^{\prime}\right\|^{-1}<\zeta
$$

$$
m\left(B\left(x, \gamma \theta^{-1} \beta K r\right)\right) \geqslant \alpha^{1 / 6}\left|\phi_{\left.\omega\right|_{n-1}}^{\prime}\left(\pi\left(\sigma^{n-1}(\omega)\right)\right)\right|^{h} m\left(B\left(\pi\left(\sigma^{n-1}(\omega)\right), \gamma \theta^{-1} \beta K r\left\|\phi_{\left.\omega\right|_{n-1}}^{\prime}\right\|^{-1}\right)\right) .
$$

Now, assuming that $\omega_{n} \notin G$ and taking into account the fact that $\pi\left(\sigma^{n-1}(\omega)\right) \in \phi_{\omega_{n}}(J)$, it follows from (3.1) applied with $\rho=\theta$, along with (3.5) and (3.6), that

$$
m\left(B\left(\pi\left(\sigma^{n-1}(\omega)\right), K r\left\|\phi_{\left.\omega\right|_{n-1}}^{\prime}\right\|^{-1}\right)\right) \leqslant \alpha m\left(B\left(\pi\left(\sigma^{n-1}(\omega)\right), \gamma \theta^{-1} \beta K r\left\|\phi_{\left.\omega\right|_{n-1}}^{\prime}\right\|^{-1}\right)\right) .
$$

Combining this along with (3.7), (3.8), and (3.10), we get

$$
m(B(x, r)) \leqslant \alpha^{1 / 3} m\left(B\left(x, \gamma \theta^{-1} \beta K r\right)\right) .
$$

Suppose finally that $\omega_{n} \in G$. It then follows from (3.5) that $\gamma \theta^{-1} \beta K r\left\|\phi_{\left.\omega\right|_{n-1}}^{\prime}\right\|^{-1} \geqslant \gamma \beta \eta$, and consequently that

$$
m\left(B\left(\pi\left(\sigma^{n-1}(\omega)\right), \gamma \theta^{-1} \beta K r\left\|\phi_{\left.\omega\right|_{n-1}}^{\prime}\right\|^{-1}\right)\right) \geqslant P .
$$

Therefore, in view of (3.6) and (3.2), we get

$$
m\left(B\left(\pi\left(\sigma^{n-1}(\omega)\right), K r\left\|\phi_{\left.\omega\right|_{n-1}}^{\prime}\right\|^{-1}\right)\right) \leqslant \alpha m\left(B\left(\pi\left(\sigma^{n-1}(\omega)\right), \gamma \theta^{-1} \beta K r\left\|\phi_{\left.\omega\right|_{n-1}}^{\prime}\right\|^{-1}\right)\right) .
$$

Now, exactly the same argument leads to (3.11). This formula and (3.9) complete the proof as $\gamma \theta^{-1} \beta K>1$.

A rather straightforward argument leads to the following slightly stronger form of Theorem 3.6, which is, however, much more convenient for further applications. 


\section{DiOPHANTINE APPROXIMATION FOR CONFORMAL MEASURES}

Theorem 3.7. Suppose that the system $S=\left\{\phi_{i}\right\}_{i \in I}$ is regular, and denote by $m$ the corresponding $h$-conformal measure. Suppose also that there exists an integer $q \geqslant 0$ and $\kappa \in(0, \min \{1$, $\operatorname{dist}(X, \partial V)\})$ such that the following three conditions are satisfied.

(a) There exist three real constants $\gamma \geqslant 1, \beta>1, \alpha \in(0,1)$, and a finite set $F \subset I$ such that, for all $i \in I \backslash F$, there exists $y_{i} \in \phi_{i}(X)$ such that, for all $r \in\left[\gamma\left\|\phi_{i}^{\prime}\right\|, \kappa\right]$,

$$
m\left(B\left(y_{i}, r\right)\right) \leqslant \alpha \mu\left(B\left(y_{i}, \beta r\right)\right) .
$$

(b) For all $\omega \in I^{*}$ with $|\omega| \leqslant q$, all $x \in J$, and all $r \in(0, \kappa)$,

$$
m\left(\phi_{\omega}(B(x, r))\right) \leqslant \alpha^{-1 / 3}\left|\phi_{\omega}^{\prime}(x)\right|^{h} m(B(x, r)) .
$$

(c) For all $\omega \in I^{q}$ and all $x \in J$,

$$
\phi_{\omega}(B(x, \kappa)) \subset X .
$$

Then the $h$-conformal measure $m$ satisfies the (efd) property.

Proof. In view of Theorem 3.6 it suffices to check condition (a) of this theorem with appropriate $\kappa>0$ (smaller) and $\beta>1$ (larger). All other constants will remain unchanged. Indeed, for every $i \in I$, every $r \geqslant \gamma\left\|\phi_{i}^{\prime}\right\|$, and every $x \in \phi_{i}(X)$, we have

$$
B(x, r) \subset B\left(y_{i}, r+\operatorname{diam}\left(\phi_{i}(X)\right)\right) \subset B\left(y_{i}, r+D\left\|\phi_{i}^{\prime}\right\|\right) \subset B\left(y_{i}, r+\frac{D}{\gamma} r\right)=B\left(y_{i},\left(1+\frac{D}{\gamma}\right) r\right) .
$$

Putting $\beta^{\prime}=\beta(1+(D / \gamma) r)+(D / \gamma)>\beta>1$, we get

$$
\begin{aligned}
B\left(x, \beta^{\prime} r\right) & \supset B\left(y_{i}, \beta^{\prime} r-\operatorname{diam}\left(\phi_{i}(X)\right)\right) \supset B\left(y_{i}, \beta^{\prime} r-D\left\|\phi_{i}^{\prime}\right\|\right) \supset B\left(y_{i}, \beta^{\prime} r-\frac{D}{\gamma} r\right) \\
& =B\left(y_{i},\left(\beta^{\prime}-\frac{D}{\gamma}\right) r\right)=B\left(y_{i}, \beta\left(1+\frac{D}{\gamma}\right) r\right) .
\end{aligned}
$$

Now assuming in addition that $i \in I \backslash F$ and that $r \in(0,(1+(D / \gamma)) \kappa)$, we therefore obtain from condition (a) that

$$
m(B(x, r)) \leqslant m\left(B\left(y_{i},\left(1+\frac{D}{\gamma}\right) r\right)\right) \leqslant \alpha m\left(B\left(y_{i}, \beta\left(1+\frac{D}{\gamma}\right) r\right)\right) \leqslant \alpha m\left(B\left(x, \beta^{\prime} r\right)\right) .
$$

We are done.

Thinking about applications of Theorem 3.7, let us prove the following.

Proposition 3.8. If $S=\left\{\phi_{i}: X \rightarrow X\right\}_{i \in I}$ is a regular conformal iterated function system satisfying the SSOSC, then the conditions (b) and (c) of Theorem 3.7 are satisfied.

Proof. Condition (c) of Theorem 3.7 is satisfied by taking $q=0$ and any positive $\kappa<\operatorname{dist}(J, \partial X)$. Condition (b) of Theorem 3.7 is then obviously satisfied.

\section{Real continued fractions: general results}

In this section we examine the efd property of conformal measures of continued fractions iterated function systems with restricted entries. For every $n \geqslant 1$ we consider the map $\phi_{n}:[0,1] \rightarrow[0,1]$ given by the formula

$$
\phi_{n}(x)=\frac{1}{x+n} .
$$

If $I \subset \mathbb{N}$ contains at least two points, we call $S_{I}=\left\{\phi_{n}\right\}_{n \in I}$ a continued fraction iterated function system. It is straightforward to see that the limit set of this system consists of all irrational numbers 


\section{URBAŃSKI}

whose continued fraction expansion has all entries in $I$. Many geometrical properties of such limit sets have been thoroughly investigated in [MU99, Urb01a, MU03b]. We start our considerations by recalling the following result proven in [MU03b] as Lemma 3.2.

Proposition 4.1. If $1 \notin I$ and $S=\left\{\phi_{i}\right\}_{i \in I}$ is a regular continued fraction iterated function system, then $S$ satisfies the SSOSC, and consequently, due to Proposition 3.8, the conditions (b) and (c) of Theorem 3.7 are satisfied.

In order to get rid of the rather restrictive, fairly artificial, and irritating assumption that $1 \notin I$, we shall prove the following.

Lemma 4.2. Assume that $S=\left\{\phi_{i}\right\}_{i \in I}$ is a regular continued fraction iterated function system and let $m$ be the corresponding $h$-conformal measure. If

$$
\limsup _{n \rightarrow \infty}\left\{m\left(\left[\frac{1}{n+1}, \frac{1}{n}\right]\right) / m\left(\left[0, \frac{1}{n+1}\right]\right)\right\}<\infty,
$$

then there exist $\alpha \in(0,1)$ and $\kappa>0$ such that the conditions (b) and (c) of Theorem 3.7 are satisfied with $q=2$.

Proof. Fix $\kappa \in(0,1 / 3)$. We first verify condition (c) of Theorem 3.7. For every $x \in[1 / 2,1]$ and every $n \geqslant 1$, we have

$$
\phi_{n}(B(x, \kappa))=\left(\frac{1}{n+x+\kappa}, \frac{1}{n+x-\kappa}\right) \subset(0,1) .
$$

If $x \in[0,1 / 2]$, then

$$
\phi_{n}(B(x, \kappa))=\left(\frac{1}{n+x+\kappa}, \frac{1}{n+x-\kappa}\right) \subset(0, \infty)
$$

and therefore, for every $k \geqslant 1$,

$$
\phi_{k n}(B(x, \kappa)) \subset \phi_{k}((0, \infty))=(0,1 / k) \subset(0,1) .
$$

(here $k n$ is the concatenation of the letters $k$ and $n$, and not their product). Hence the condition

(c) of Theorem 3.7 is satisfied with $q=2$.

In order to verify condition (b) of Theorem 3.7 put

$$
B(z, r, R)=\{x \in \mathbb{R}: z-r \leqslant x \leqslant z+R\} .
$$

The proof is split into two cases and several subcases.

Case 1: assume $x \in(1 / 2,1] \cap J$ and fix $r \in(0, \kappa]$. Without loss of generality we may assume that $x+r>1$. Fix $R \in[r, 4 r]$. Then for every $n \in I$,

$$
\phi_{n}(B(x, r, R))=\phi_{n}((x-r, 1]) \cup \phi_{n}([1, x+R)) .
$$

Now

$$
\begin{aligned}
m\left(\phi_{n}((x-r, 1])\right) & \leqslant\left(\frac{n+x}{n+x-r}\right)^{2 h}\left(\frac{1}{n+x}\right)^{2 h} m((x-r, 1]) \\
& =\left(\frac{1}{1-r /(n+x)}\right)^{2 h}\left|\phi_{n}^{\prime}(x)\right|^{h} m((x-r, 1]) \leqslant 2\left|\phi_{n}^{\prime}(x)\right|^{h} m((x-r, 1]), \\
& =2\left|\phi_{n}^{\prime}(x)\right|^{h} m(B(x, r, R))
\end{aligned}
$$

where the last inequality sign was written assuming that $\kappa>0$ (and consequently $r<\kappa$ ) is small enough. Also

$$
\phi_{n}([1, x+R))=\left(\frac{1}{n+x+R}, \frac{1}{n+1}\right]=\phi_{n+1}([0, x+R-1)) .
$$




\section{DiOPHANTINE APPROXIMATION FOR CONFORMAL MEASURES}

Hence (note that if $n+1 \notin I$, then $\left.m\left(\phi_{n}([1, x+R))\right)=m\left(\phi_{n+1}([0, x+R-1))\right)=0\right)$,

$$
\begin{aligned}
m\left(\phi_{n}([1, x+R))\right) & \leqslant\left(\frac{1}{n+1}\right)^{2 h} m([0, x+R-1))=\left(\frac{n+x}{n+1}\right)^{2 h}\left(\frac{1}{n+x}\right)^{2 h} m([0, x+R-1)) \\
& \leqslant(n+x)^{-2 h} m([0, x+R-1))=\left|\phi_{n}^{\prime}(x)\right|^{h} m([0, x+R-1)) .
\end{aligned}
$$

Now, $(x-r, 1]=\phi_{1}\left(\left[0,(x-r)^{-1}-1\right)\right)$, and consequently $(x \in(1 / 2,1] \cap J$ implies that $1 \in I)$

$$
m((x-r, 1]) \geqslant\left(\frac{1}{1+0}\right)^{2 h} m\left(\left[0,(x-r)^{-1}-1\right)\right)=m\left(\left[0,(x-r)^{-1}-1\right)\right) .
$$

Since it is straightforward to verify that $x+R-1 \leqslant(x-r)^{-1}-1(r<\kappa<1 / 3)$, combining (4.3) and (4.4), we get

$m\left(\phi_{n}([1, x+R))\right) \leqslant\left|\phi_{n}^{\prime}(x)\right|^{h} m\left(\left[0,(x-r)^{-1}-1\right)\right) \leqslant\left|\phi_{n}^{\prime}(x)\right|{ }^{h} m((x-r, 1])=\left|\phi_{n}^{\prime}(x)\right|^{h} m(B(x, r, R))$.

Combining this with (4.2) and (4.1) in turn, we obtain

$$
m\left(\phi_{n}(B(x, r, R))\right) \leqslant 3\left|\phi_{n}^{\prime}(x)\right|^{h} m(B(x, r, R)),
$$

which finishes the proof in Case 1.

Before moving to the next case, observe first that, due to our assumptions,

$$
Q:=\max \left\{1, \sup _{j \geqslant 1}\left\{\frac{m\left(\left[0, j^{-1}\right)\right)}{m\left(\left[0,(j+1)^{-1}\right)\right)}\right\}\right\}<\infty .
$$

Case 2: suppose that $x \in[0,1 / 2]$. We may assume without loss of generality that $x-r<0$. Then, for every $n \in I$,

$$
\phi_{n}(B(x, r, R))=\phi_{n}((x-r, 0]) \cup \phi_{n}((0, x+R)) .
$$

Now,

$$
\begin{aligned}
m\left(\phi_{n}((0, x+R))\right) & \leqslant\left(\frac{1}{n}\right)^{2 h} m((0, x+R))=\left(\frac{n+x}{n}\right)^{2 h}\left(\frac{1}{n+x}\right)^{2 h} m((0, x+R)) \\
& =\left(1+\frac{x}{n}\right)^{2 h}\left|\phi_{n}^{\prime}(x)\right|^{h} m((0, x+R)) \leqslant 2^{h}\left|\phi_{n}^{\prime}(x)\right|^{h} m((0, x+R)) \\
& \leqslant 2\left|\phi_{n}^{\prime}(x)\right|^{h} m(B(x, r, R)) .
\end{aligned}
$$

Case 2(a): suppose first that $n=1$. Then $\phi_{1}((x-r, 0])=\left[1,(1+x-r)^{-1}\right) \subset[1, \infty)$. Thus, $m\left(\phi_{1}((x-r, 0])\right)=0$, and combining this with (4.6) and (4.7), we obtain

$$
m\left(\phi_{1}(B(x, r, R))\right) \leqslant 2\left|\phi_{1}^{\prime}(x)\right|^{h} m(B(x, r, R)) .
$$

Case 2(b): assume that $n \geqslant 2$. Then

$$
\phi_{n}((x-r, 0])=\left[\frac{1}{n}, \frac{1}{n+x-r}\right)=\phi_{n-1}((x-r+1,1]),
$$

and therefore (note that if $n-1 \notin I$, then $m\left(\phi_{n}((x-r, 0])=m\left(\phi_{n-1}((x-r+1,1])\right)=0\right)$

$$
\begin{aligned}
m\left(\phi_{n}((x-r, 0])\right) & \leqslant\left(\frac{1}{n-1+(x-r+1)}\right)^{2 h} m((x-r+1,1]) \\
& =\left(\frac{n+x}{n+x-r}\right)^{2 h}\left(\frac{1}{n+x}\right)^{2 h} m((x-r+1,1]) \\
& =\left(1+\frac{r}{n+x-r}\right)\left|\phi_{n}^{\prime}(x)\right|^{h} m((x-r+1,1]) \\
& \leqslant 2\left|\phi_{n}^{\prime}(x)\right|^{h} m((x-r+1,1]) .
\end{aligned}
$$




\section{URBAŃSKI}

Since $(x-r+1,1]=\phi_{1}\left(\left[0,(x-r+1)^{-1}-1\right)\right)$, we get $($ as above if $1 \notin I$, then $m((x-r+1,1])=0)$

$$
m((x-r+1,1]) \leqslant\left(\frac{1}{1+0}\right)^{2 h} m\left(\left[0,(x-r+1)^{-1}-1\right)\right)=m\left(\left[0,(x-r+1)^{-1}-1\right)\right) .
$$

Now, taking $\kappa>0$ small enough (and consequently $r$ and $r-x$ small enough), we see that $(x-r+$ $1)^{-1}-1=(1-(r-x))^{-1}-1 \leqslant r-x+2(r-x)^{2} \leqslant r-x+2 r^{2}$. Picking an integer $k \geqslant 1$ such that

$$
\frac{1}{k+1}<x+r \leqslant \frac{1}{k}
$$

we therefore get

$$
\frac{1}{x-r+1}-1-\frac{1}{k} \leqslant \frac{1}{x-r+1}-1-(x+r) \leqslant r-x+2 r^{2}-x-r=2 r^{2}-2 x \leqslant 2 r^{2} \leqslant \frac{2}{k^{2}} .
$$

Consider now two subcases.

Case 2(b)(1): $k \geqslant 3$. It then follows from the last formula that $(x-r+1)^{-1}-1<(k-2)^{-1}$. Therefore, using (4.11), we get

$$
\begin{aligned}
\frac{m\left(\left[0,(x-r+1)^{-1}-1\right)\right)}{m([0, x+r))} & \leqslant \frac{m\left(\left[0,(k-2)^{-1}\right)\right)}{m\left(\left[0,(k+1)^{-1}\right)\right)} \\
& =\frac{m\left(\left[0,(k-2)^{-1}\right)\right)}{m\left(\left[0,(k-1)^{-1}\right)\right)} \frac{m\left(\left[0,(k-1)^{-1}\right)\right)}{m\left(\left[0, k^{-1}\right)\right)} \frac{m\left(\left[0, k^{-1}\right)\right)}{m\left(\left[0,(k+1)^{-1}\right)\right)} \\
& \leqslant Q^{3} .
\end{aligned}
$$

Case 2(b)(2): $k \leqslant 2$. Then similarly

$$
\frac{m\left(\left[0,(x-r+1)^{-1}-1\right)\right)}{m([0, x+r))} \leqslant \frac{m([0,1))}{m([0,1 / 3))} \leqslant Q^{2} .
$$

The rest of the proof is performed for all $k \geqslant 1$ without distinguishing between Case 2(b)(1) and Case 2(b)(2). Combining (4.13) with (4.12), (4.10), and (4.9), we obtain

$$
\begin{aligned}
m\left(\phi_{n}((x-r, 0])\right) & \leqslant 2 Q^{3}\left|\phi_{n}^{\prime}(x)\right|^{h} m([0, x+r)) \leqslant 2 Q^{3}\left|\phi_{n}^{\prime}(x)\right|^{h} m([0, x+R)) \\
& =2 Q^{3}\left|\phi_{n}^{\prime}(x)\right|^{h} m(B(x, r, R)) .
\end{aligned}
$$

Looking at (4.7) and (4.6), we therefore get

$$
m\left(\phi_{n}(B(x, r, R))\right) \leqslant 2\left(1+Q^{3}\right)\left|\phi_{n}^{\prime}(x)\right|^{h} m(B(x, r, R)) .
$$

Combining this estimate with (4.8) and (4.5), we see (note that $Q \geqslant 1$ ) that (4.14) is true for all $n \geqslant 1$, all $\kappa \in(0,1 / 3)$ small enough, all $r \in(0, \kappa)$, all $R \in[r, 4 r]$, and all $x \in J$. In particular,

$$
m\left(\phi_{n}(B(x, r))\right) \leqslant 2\left(1+Q^{3}\right)\left|\phi_{n}^{\prime}(x)\right|^{h} m(B(x, r)) .
$$

Now keep $x \in J$ and $r \in(0, \kappa)$. Take any two numbers $k, n \in I$. Since $\phi_{n}(B(x, r))=B\left(\phi_{n}(x)\right.$, $\left.r_{n}, R_{n}\right)$, where $r_{n} \geqslant r(n+1)^{-2}$ and $R_{n} \leqslant r n^{-2}$ (so $\left.R_{n} / r_{n} \leqslant((n+1) / n)^{2} \leqslant 4\right)$, the formula (4.14) applies, and we get

$$
m\left(\phi_{k n}(B(x, r))\right)=m\left(\phi_{k}\left(B\left(\phi_{n}(x), r_{n}, R_{n}\right)\right)\right) \leqslant 2\left(1+Q^{3}\right)\left|\phi_{k}^{\prime}\left(\phi_{n}(x)\right)\right|^{h} m\left(B\left(\phi_{n}(x), r_{n}, R_{n}\right)\right) .
$$

Applying (4.14) once more, we obtain that

$$
m\left(B\left(\phi_{n}(x), r_{n}, R_{n}\right)\right)=m\left(\phi_{n}(B(x, r))\right) \leqslant 2\left(1+Q^{3}\right)\left|\phi_{n}^{\prime}(x)\right|^{h} m(B(x, r)) .
$$

Combining the last two estimates together, we finally get

$$
m\left(\phi_{k n}(B(x, r))\right) \leqslant 4\left(1+Q^{3}\right)^{2} \mid \phi_{k n}^{\prime}(x) m(B(x, r)) .
$$

This and (4.15) complete the proof. 


\section{DiOPHANTINE APPROXIMATION FOR CONFORMAL MEASURES}

Let us now recall from [MU03b] that, given $\delta \in[0,1]$ and $g>1$, we call an infinite subset $I$ of $\mathbb{N}(\delta, g)$-evenly distributed provided that there exist constants $A \geqslant 1$ and $p \geqslant 1$ such that, for all integers $n \geqslant 1$,

$$
\#(I \cap[n, g n]) \leqslant A n^{\delta},
$$

and if, in addition, $n \in I \cap[p, \infty)$, then

$$
\min \left\{\#\left(I \cap[n, g n], \#\left(I \cap\left[g^{-1} n, n\right]\right)\right\} \geqslant A^{-1} n^{\delta} \geqslant 2 .\right.
$$

If we do not want (do not need) to specify $\delta$ and $g$, we simply say that $I$ is evenly distributed. A simple, straightforward but useful property of $(\delta, g)$-evenly distributed sets is contained in the following.

Lemma 4.3. If $I \subset \mathbb{N}$ is a $(\delta, g)$-evenly distributed set, then for all $n \in \mathbb{N}$ large enough, $I \cap[n, g n] \neq \emptyset$ and $I \cap\left[g^{-1} n, n\right] \neq \emptyset$, and consequently

$$
\min \left\{\#\left(I \cap\left[n, g^{2} n\right], \#\left(I \cap\left[g^{-2} n, n\right]\right)\right\} \geqslant A^{-1} g^{-2 \delta} n^{\delta} .\right.
$$

We say that an infinite subset $I$ of $\mathbb{N}$ is rapidly growing if for every $\xi>0$ there exist $u \geqslant p$ and $\sigma>1$ such that, for every $n \in I \cap[u, \infty)$ and every $k \in[1, n / 7]$,

$$
\#(I \cap([n-\sigma k, n-k] \cup[n+k, n+\sigma k])) \geqslant \xi \#(I \cap[n-k, n+k]) .
$$

We have proved in [MU03b] the following result as Proposition 3.3.

Proposition 4.4. If $I \subset \mathbb{N}$ is a $(\delta, g)$-evenly distributed set, then the system $S_{I}$ is cofinitely (hereditarily) regular and $\theta_{S}=\delta / 2$. Consequently, $h>\delta / 2$.

The main result of this section is the following.

THEOREM 4.5. If $I \subset \mathbb{N}$ is a rapidly growing evenly distributed set, then the corresponding conformal measure $m$ satisfies the efd property, and is consequently extremal due to Theorem 3.2.

Proof. Our general strategy is to verify the assumptions of Theorem 3.7. The first step is to verify the assumptions of Lemma 4.2. Indeed, observe that if $n \notin I$, then

$$
m([1 /(n+1), 1 / n])=0,
$$

and if $n \in I$, then

$$
m\left(\left[\frac{1}{n+1}, \frac{1}{n}\right]\right) \leqslant n^{-2 h} .
$$

If in addition $n \geqslant p$, then by (4.17) there exists $k \in I \cap[n+1, g n)$. We then have

$$
m\left(\left(0, \frac{1}{n+1}\right]\right) \geqslant m\left(\left[\frac{1}{k+1}, \frac{1}{k}\right]\right) \geqslant(k+1)^{-2 h} \geqslant(2 g n)^{-2 h}=(2 g)^{-2 h} n^{-2 h} .
$$

Combining this with (4.19) and (4.20), we see that for all $n \in I \cap[p, \infty)$,

$$
m\left(\left[\frac{1}{n+1}, \frac{1}{n}\right]\right) / m\left(\left(0, \frac{1}{n+1}\right]\right) \leqslant(2 g)^{2 h} .
$$

Thus, the assumptions of Lemma 4.2 are satisfied, and applying it we see that, due to Theorem 3.7, it suffices to verify the following condition.

For every $\alpha \in(0,1)$ there exist $\beta>1$ and a finite set $F \subset I$ such that, for all $n \in I \backslash F$ and all $r \in\left(1 / n^{2}, 1 / 3\right)$, we have

$$
m(B(1 / n, r)) \leqslant \alpha m(B(1 / n, \beta r))
$$




\section{URBAŃSKI}

For each $r>0$ put

$$
I_{-}(r)=I \cap\left\{k \leqslant n: \frac{1}{k}-\frac{1}{n}<r\right\}=I \cap\left[\frac{n}{1+r n}, n\right] .
$$

For every $r \in(0,1 / n)$ put

$$
I_{+}(r)=I \cap\left\{k \geqslant n: \frac{1}{n}-\frac{1}{k}<r\right\}=I \cap\left[n, \frac{n}{1-r n}\right]
$$

and for $r \geqslant 1 / n$ put

$$
I_{+}(r)=I \cap[n,+\infty) .
$$

Also set

$$
I(r)=I_{+}(r) \cup I_{-}(r) .
$$

Since the symmetric difference of $J \cap B(x, r)$ and $\bigcup_{k \in I(r)} \phi_{k}(J)$ is contained in $\phi_{\inf I_{-}(r)-1}(J) \cup$ $\phi_{\text {sup } I_{+}(r)}(J)$, we easily deduce from Lemma 4.3 that

$$
m(B(1 / n, r)) \asymp \sum_{k \in I(r)} m\left(\phi_{k}(J)\right) \asymp \sum_{k \in I(r)} k^{-2 h} \asymp \sum_{k \in I_{+}(r)} k^{-2 h}+\sum_{k \in I_{-}(r)} k^{-2 h} .
$$

Now take an arbitrary $\xi>0$ and fix $u \geqslant p \geqslant 2$ and $\sigma \geqslant 3$ so that the condition (4.18) is satisfied. The assumptions of our theorem imply that $I$ is $(\delta, g)$-evenly distributed with some $\delta \in(0,1]$ and some $g>1$. Fix $n \in I \cap[u, \infty)$ and consider the following cases.

Case 1: $r \in[1 / 4(\sigma+1) n, 1 / 3]$. Using Lemma 4.3, we get for all $n \in I \cap[u, \infty)$ sufficiently large that

$$
\begin{aligned}
\sum_{k \in I_{-}(r)} k^{-2 h} & =\sum_{k=n /(1+r n)}^{n} k^{-2 h} \asymp \sum_{j=0}^{\log _{g^{2}}(1+r n)}\left(n g^{-2 j}\right)^{-2 h+\delta}=n^{\delta-2 h} \sum_{j=0}^{\log _{g^{2}}(1+r n)} g^{2(2 h-\delta) j} \\
& \asymp n^{\delta-2 h} \frac{g^{2(2 h-\delta) \log _{g^{2}}(1+r n)}-1}{g^{2(2 h-\delta)}-1} \asymp n^{\delta-2 h} g^{2(2 h-\delta) \log _{g^{2}}(1+r n)} \\
& =n^{\delta-2 h}(1+r n)^{2 h-\delta} \in\left[n^{\delta-2 h}(r n)^{2 h-\delta}, n^{\delta-2 h}((4 \sigma+5) r n)^{2 h-\delta}\right] \\
& =\left[r^{2 h-\delta},\left(\frac{4 \sigma+5}{4(\sigma+1)}\right)^{2 h-\delta} r^{2 h-\delta}\right] \subset\left[r^{2 h-\delta}, 4 r^{2 h-\delta}\right] .
\end{aligned}
$$

Therefore, using (4.22), we get that

$$
m\left(B\left(1 / n, \sigma^{3} r\right)\right) \geqslant \sum_{k \in I_{-}\left(\sigma^{3} r\right)} k^{-2 h} \succeq \sigma^{3(2 h-\delta)} r^{2 h-\delta} .
$$

Also

$$
\begin{aligned}
\sum_{k=n}^{\infty} k^{-2 h} & \asymp \sum_{j=0}^{\infty}\left(n g^{2 j}\right)^{\delta-2 h}=n^{\delta-2 h} \sum_{j=0}^{\infty} g^{(\delta-2 h) j} \asymp n^{\delta-2 h} \frac{1}{1-g^{\delta-2 h}} \asymp\left(\frac{1}{n}\right)^{2 h-\delta} \\
& \leqslant(4(\sigma+1))^{2 h-\delta} r^{2 h-\delta} .
\end{aligned}
$$

Combining this equation, (4.23) and (4.22), we obtain

$$
m(B(1 / n, r)) \preceq 4^{2 h-\delta} r^{2 h-\delta}+(4(\sigma+1))^{2 h-\delta} r^{2 h-\delta} \leqslant(4(\sigma+2))^{2 h-\delta} r^{2 h-\delta} .
$$

Combining this with (4.24), we get

$$
m\left(B\left(1 / n, \sigma^{3} r\right)\right) \succeq \sigma^{2 h-\delta} m(B(1 / n, r))
$$

for all $\sigma \geqslant 3$ large enough. 


\section{DiOPHANTINE APPROXIMATION FOR CONFORMAL MEASURES}

Case 2: $r \in\left[1 / n^{2}, 1 / 4(\sigma+1) n\right]$. Then

$$
1 \geqslant \frac{n}{1+r n} \cdot \frac{1}{n}=\frac{1}{1+r n} \geqslant \frac{1}{1+1 / 4(\sigma+1)} \geqslant \frac{1}{2} .
$$

Therefore,

$$
\sum_{j \in I_{-}(r)} j^{-2 h}=\sum_{j=n /(1+r n)}^{n} j^{-2 h} \asymp n^{-2 h} \#\left(I \cap\left[\frac{n}{1+r n}, n\right]\right)
$$

and

$$
\sum_{j \in I_{-}(2 \sigma r) \backslash I_{-}(r)} j^{-2 h}=\sum_{j=n /(1+2 \sigma r n)}^{n /(1+r n)} j^{-2 h} \succeq n^{-2 h} \#\left(I \cap\left[\frac{n}{1+2 \sigma r n}, \frac{n}{1+r n}\right]\right) .
$$

Since $r n \leqslant 1 / 4(\sigma+1)$, we get

$$
1 \leqslant \frac{n}{1-r n} \cdot \frac{1}{n} \leqslant \frac{1}{1-1 /[4(\sigma+1)]} \leqslant 2
$$

and therefore

$$
\sum_{j \in I_{+}(r)} j^{-2 h}=\sum_{j=n}^{n /(1-r n)} \asymp n^{-2 h} \#\left(I \cap\left[n, \frac{n}{1-r n}\right]\right) .
$$

Similarly

$$
1 \leqslant \frac{n}{1-2 \sigma r n} \cdot \frac{1}{n} \leqslant \frac{1}{1-2 \sigma /[4(\sigma+1)]}=\frac{1}{1-\sigma /[2(\sigma+1)]}=\frac{2(\sigma+1)}{\sigma+2} \leqslant 2,
$$

and therefore

$$
\sum_{j \in I_{+}(2 \sigma r) \backslash I_{+}(r)} j^{-2 h}=\sum_{j=n /(1-r n)}^{n /(1-2 \sigma r n)} j^{-2 h} \asymp n^{-2 h} \#\left(I \cap\left[\frac{n}{1-r n}, \frac{n}{1-2 \sigma r n}\right]\right) .
$$

Combining this and (4.27), we get

$$
\sum_{j \in I(2 \sigma r) \backslash I(r)} j^{-2 h} \succeq n^{-2 h} \#\left(I \cap\left(\left[\frac{n}{1+2 \sigma r n}, \frac{n}{1+r n}\right] \cup\left[\frac{n}{1-r n}, \frac{n}{1-2 \sigma r n}\right]\right)\right),
$$

and combining (4.26) with (4.28), we obtain

$$
\sum_{j \in I(r)} j^{-2 h} \asymp n^{-2 h} \#\left(I \cap\left[\frac{n}{1+r n}, \frac{n}{1-r n}\right]\right) .
$$

Now put

$$
k=\frac{n}{1-r n}-n=\frac{r n^{2}}{1-r n} .
$$

Since $r \geqslant 1 / n^{2}$, we get

$$
k \geqslant \frac{n}{1-1 / n}-n=\frac{n}{n-1}>1 .
$$

Since $r n \leqslant 1 / 4(\sigma+1)$, we obtain

$$
k \leqslant \frac{n}{1-1 /[4(\sigma+1)]}-n=n \frac{1}{4 \sigma+3} \leqslant \frac{n}{7} .
$$

We now want to check that

$$
n+\sigma k \leqslant \frac{n}{1-2 \sigma r n} .
$$




\section{URBAŃSKI}

This equivalently means that $-2 \sigma r n^{2}+\sigma k(1-2 \sigma r n) \leqslant 0$ or

$$
\frac{r n^{2}}{1-r n}(1-2 \sigma r n) \leqslant 2 r n^{2} \Leftrightarrow \frac{1-2 \sigma r n}{1-r n} \leqslant 2
$$

and since this last inequality is obviously true, so is (4.33). Hence,

$$
I \cap[n, n+\sigma k] \subset I \cap\left[n, \frac{n}{1-2 \sigma r n}\right] .
$$

We now want to check that

$$
n-\sigma k \geqslant \frac{n}{1+2 \sigma r n} .
$$

This equivalently means that $2 \sigma r n^{2}-\sigma k(1+2 \sigma r n) \geqslant 0$ or (recall that $k=r n^{2} /(1-r n)$ )

$$
\frac{r n^{2}}{1-r n}(1+2 \sigma r n) \leqslant 2 r n^{2} \Leftrightarrow \frac{1+2 \sigma r n}{1-r n} \leqslant 2 \Leftrightarrow 1+2 \sigma r n \leqslant 2-2 r n \Leftrightarrow 2(\sigma+1) r n \leqslant 1
$$

and this last inequality is true because of our definition of Case 2. Hence (4.35) is satisfied, and we get

Along with (4.34) this gives that

$$
I \cap[n-\sigma k, n] \subset I \cap\left[\frac{n}{1+2 \sigma r n}, n\right] .
$$

$$
I \cap[n-\sigma k, n+\sigma k] \subset I \cap\left[\frac{n}{1+2 \sigma r n}, \frac{n}{1-2 \sigma r n}\right] .
$$

We also need to check that

$$
n-k \leqslant \frac{n}{1+r n} .
$$

But this equivalently means that $r n^{2}-k(1+r n) \leqslant 0$ or

$$
k \geqslant \frac{r n^{2}}{1+r n} \Leftrightarrow \frac{r n^{2}}{1-r n} \geqslant \frac{r n^{2}}{1+r n} \Leftrightarrow 1+r n \geqslant 1-r n .
$$

This last inequality is obviously true and (4.37) is verified. Hence

$$
I \cap[n-k, n+k] \supset I \cap\left[\frac{n}{1+r n}, \frac{n}{1-r n}\right] .
$$

As an immediate consequence of this and (4.36), we obtain

$$
I \cap\left(\left[\frac{n}{1+2 \sigma r n}, \frac{n}{1+r n}\right] \cup\left[\frac{n}{1-r n}, \frac{n}{1-2 \sigma r n}\right]\right) \supset I \cap([n-\sigma k, n-k] \cup[n+k, n+\sigma k]) .
$$

Looking now at (4.29), (4.38), (4.18) (which applies due to (4.31) and (4.32)), (4.37), the definition of $k$, and (4.30), we get

$$
\begin{aligned}
\sum_{j \in I(2 \sigma r)} j^{-2 h} & =\sum_{j \in I(r)} j^{-2 h}+\sum_{j \in I(2 \sigma r) \backslash I(r)} j^{-2 h} \geqslant \sum_{j \in I(2 \sigma r) \backslash I(r)} j^{-2 h} \\
& \geqslant n^{-2 h} \#\left(I \cap\left(\left[\frac{n}{1+2 \sigma r n}, \frac{n}{1+r n}\right] \cup\left[\frac{n}{1-r n} \frac{n}{1-2 \sigma r n}\right]\right)\right) \\
& \geqslant n^{-2 h} \#(I \cap([n-\sigma k, n-k] \cup[n+k, n+\sigma k])) \geqslant \xi n^{-2 h} \#(I \cap([n-k, n+k])) \\
& \geqslant \xi n^{-2 h} \#\left(I \cap\left[\frac{n}{1+r n}, \frac{n}{1-r n}\right]\right) \asymp \xi \sum_{j \in I(r)} j^{-2 h} \\
& \asymp \xi m(B(1 / n, r)) .
\end{aligned}
$$




\section{DiOPHANTINE APPROXIMATION FOR CONFORMAL MEASURES}

Thus, for $\sigma>1$ large enough, we have

$$
m\left(B\left(1 / n, \sigma^{3} r\right)\right) \geqslant m(B(1 / n, 2 \sigma r)) \geqslant \xi m(B(1 / n, r)) .
$$

Along with (4.25) this implies that, for all $\xi$ (and consequently $\sigma$ ) large enough, all $n \in I$ large enough, and for every $r \in\left[\left\|\phi_{n}^{\prime}\right\|, 1 / 3\right]$, we have

$$
m\left(B\left(1 / n, \sigma^{3} r\right)\right) \geqslant \min \left\{\xi, \sigma^{2 h-\delta}\right\} m(B(1 / n, r)) .
$$

Letting $\xi \nearrow+\infty$ (and consequently $\sigma \nearrow+\infty$ ), we see that $(4.21)$ is verified. We are done.

\section{Real continued fractions: examples}

Represent any set $I \subset \mathbb{N}$ as a non-decreasing sequence $\left\{a_{n}\right\}_{n=1}^{\infty}$. Recall that $I$ is said to have bounded gaps if $\sup _{n \geqslant 1}\left\{a_{n+1}-a_{n}\right\}<\infty$. Our first result in this section is the following.

TheOREM 5.1. If $I \subset \mathbb{N}$ has bounded gaps, then the continued fraction iterated function system $S_{I}$ is regular and the corresponding conformal measure (which due to Corollary 5.9 from [MU99] is, up to a multiplicative constant, equal to the $h$-dimensional packing measure on $J_{S}$ ) satisfies the efd property, and is consequently extremal due to Theorem 3.2.

Proof. It is obvious that $I$ is $(1, g)$-evenly distributed for all $g>1$ large enough. Since

$$
\lim _{\sigma \rightarrow \infty}\left(\inf _{n \geqslant 1} \inf _{k \geqslant 1}\left\{\frac{\#(I \cap[n+k, n+\sigma k])}{2 k}\right\}\right)=\infty
$$

we see that the set $I$ is rapidly growing. Thus, invoking Theorem 4.5 completes the proof.

Corollary 5.2. If $I \subset \mathbb{N}$ is an arithmetic progression, then the corresponding conformal measure satisfies the efd property and is extremal.

As was noticed in Theorem 5.1, the $h$-conformal measure of any system with bounded gaps is, up to a multiplicative constant, equal to the $h$-dimensional packing measure on $J_{S}$. It follows from Corollary 4.5 in [MU99] that if in addition $I$ is a proper infinite subset of $\mathbb{N}$, then the $h$-dimensional Hausdorff measure on $J_{S}$ vanishes.

Lemma 5.3. Suppose that $\left\{x_{n}\right\}_{n=1}^{\infty}$, an unbounded increasing sequence of positive integers, and $\left\{d_{n}\right\}_{n=1}^{\infty}$, a non-decreasing sequence of positive integers, satisfy the following conditions:

$$
x_{n}+d_{n}<\frac{6}{7}\left(x_{n+1}-d_{n+1}\right) ;
$$

(b) for every $j \geqslant 1$

$$
\liminf _{n \rightarrow \infty} \frac{d_{n}}{x_{n+j}-x_{n}+d_{n+j}}>0 .
$$

Then the set

$$
I=\bigcup_{n \geqslant 1}\left[x_{n}-d_{n}, x_{n}+d_{n}\right]
$$

is rapidly growing.

Proof. Fix an integer $\xi \geqslant 1$. In view of (b),

$$
\theta=\theta_{\xi}:=\inf _{n \geqslant 1}\left\{\frac{d_{n}}{x_{n+\xi}-x_{n}+d_{n+\xi}}\right\}>0 .
$$

Put

$$
\sigma=(2 \xi+1)\left(1+\theta^{-1}\right)
$$




\section{URBAŃSKI}

Take $n \geqslant 2, l \in\left[x_{n}-d_{n}, x_{n}+d_{n}\right]$, and $k \in[1, l / 7]$. Consider separately two cases: Case $1, k \leqslant$ $(2 \xi+1)^{-1} d_{n}$; and Case $2, k>(2 \xi+1)^{-1} d_{n}$.

Let us deal first with the Case 1. Suppose that $l-\sigma k \geqslant x_{n}-d_{n}$. Then $[l-\sigma k, l-k] \cap I=$ $[l-\sigma k, l-k]$, and therefore

$$
\#(I \cap[l-\sigma k, l-k])=(\sigma-1) k \geqslant \xi 2 k \geqslant \xi \#(I \cap[l-k, l+k]) .
$$

So suppose that $l-\sigma k<x_{n}-d_{n}$ and $l \geqslant x_{n}$. Then $I \cap[l-\sigma k, l-k] \supset\left[x_{n}-d_{n}, l-k\right]$, and therefore

$$
\#(I \cap[l-\sigma k, l-k]) \geqslant l-k-x_{n}+d_{n} \geqslant d_{n}-k \geqslant(2 \xi+1) k-k=\xi 2 k \geqslant \xi \#(I \cap[l-k, l+k]) .
$$

If $l \leqslant x_{n}$ and $l+\sigma k \leqslant x_{n}+d_{n}$, then $I \cap[l+k, l+\sigma k]=[l+k, l+\sigma k]$ and therefore \# $(I \cap$ $[l+k, l+\sigma k])=(\sigma-1) k \geqslant 2 \xi k \geqslant \xi \#(I \cap[l-k, l+k])$. If $l \leqslant x_{n}$ and $l+\sigma k>x_{n}+d_{n}$, then $I \cap[l+k, l+\sigma k] \supset\left[l+k, x_{n}+d_{n}\right]$. Hence, \# $(I \cap[l+k, l+\sigma k]) \geqslant x_{n}+d_{n}-l-k \geqslant d_{n}-k \geqslant$ $(2 \xi+1) k-k=2 \xi k \geqslant \xi \#(I \cap[l-k, l+k])$.

So, consider Case 2 . In view of (a), we have for every $n \geqslant 1$ that

$$
l+k \leqslant \frac{8}{7} l \leqslant \frac{8}{7}\left(x_{n}+d_{n}\right)<\frac{7}{6}\left(x_{n}+d_{n}\right)<x_{n+1}-d_{n+1}
$$

and

$$
l-k \geqslant \frac{6}{7} l \geqslant \frac{6}{7}\left(x_{n}-d_{n}\right)>x_{n-1}+d_{n-1} .
$$

Hence $I \cap[l-k, l+k] \subset\left[x_{n}-d_{n}, x_{n}+d_{n}\right]$, and consequently

$$
\#(I \cap[l-k, l+k]) \leqslant 2 d_{n} .
$$

Suppose now that $l+\sigma k \geqslant x_{n+\xi}+d_{n+\xi}$. It then follows from (5.2) that

$$
I \cap[l+k, l+\sigma k] \supset I \cap\left[x_{n+1}-d_{n+1}, x_{n+\xi}+d_{n+\xi}\right] \supset \bigcup_{j=n+1}^{n+\xi}\left[x_{j}-d_{j}, x_{j}+d_{j}\right] .
$$

Hence

$$
\#(I \cap[l+k, l+\sigma k]) \geqslant 2 \sum_{j=1}^{\xi} d_{n+j} \geqslant 2 \xi d_{n} .
$$

Combining this along with (5.4), we see that

$$
\#(I \cap[l+k, l+\sigma k]) \geqslant \xi \#(I \cap[l-k, l+k])
$$

for all $n \geqslant 1$. So, suppose in turn that

$$
l+\sigma k<x_{n+\xi}+d_{n+\xi}
$$

Then $\sigma k<x_{n+\xi}+d_{n+\xi}-l \leqslant x_{n+\xi}+d_{n+\xi}-x_{n}+d_{n}$, and since we are in Case $2, \sigma(2 \xi+1)^{-1} d_{n} \leqslant$ $x_{n+\xi}-x_{n}+d_{n+\xi}+d_{n}$. Invoking the definition of $\sigma$, we therefore get $\left(1+\theta^{-1}\right) d_{n}<x_{n+\xi}-x_{n}+d_{n+\xi}+d_{n}$. Equivalently, $d_{n}<\theta\left(x_{n+\xi}-x_{n}+d_{n+\xi}\right)$, which contradicts (5.1) and finishes the proof.

Applying this lemma we can easily construct the sets $I$ with unbounded gaps whose corresponding conformal measure satisfies the efd property. Indeed, we have the following.

Theorem 5.4. There exists an infinite evenly distributed rapidly growing subset $I$ of $\mathbb{N}$ with unbounded gaps. Consequently, the corresponding conformal measure on $J_{I}$ satisfies the efd property and is consequently extremal due to Theorem 3.2.

Proof. Let $A \geqslant 4$ be an integral multiple of 4 . For every $n \geqslant 4$ put

$$
x_{n}=A^{n} \text { and } d_{n}=\frac{1}{4} A^{n} .
$$




\section{DiOPHANTINE APPROXIMATION FOR CONFORMAL MEASURES}

Set

$$
I=\bigcup_{n \geqslant 1}\left[x_{n}-d_{n}, x_{n}+d_{n}\right] .
$$

Obviously $I$ is evenly distributed. In order to verify that $I$ is rapidly growing, we shall check the assumptions of Lemma 5.3. Since $x_{n}+d_{n}=\frac{5}{4} A^{n}$ and since

$$
\frac{6}{7}\left(x_{n+1}-d_{n+1}\right)=\frac{6}{7} \cdot \frac{3}{4} A^{n+1}=\frac{9}{14} A A^{n} \geqslant \frac{36}{14} A^{n} \geqslant 2 A^{n},
$$

we see that condition (a) of Lemma 5.3 is satisfied. Since, for all $n \geqslant 1$ and all $j \geqslant 1$,

$$
x_{n+j}-x_{n}+d_{n+j}=A^{n+j}-A^{n}+\frac{1}{4} A^{n+j}=A^{n}\left(\frac{5}{4} A^{j}-1\right),
$$

we get

$$
\frac{d_{n}}{x_{n+j}-x_{n}+d_{n+j}}=\frac{\frac{1}{4} A^{n}}{A^{n}\left(\frac{5}{4} A^{j}-1\right)}=\frac{1}{5 A^{j}-4}>0 .
$$

Thus condition (b) of Lemma 5.3 also holds, and all the assumptions of Lemma 5.3 have been verified. Hence, $I$ is rapidly growing. It is therefore left to check that $I$ has unbounded gaps. To see this notice that

$$
x_{n+1}-d_{n+1}-\left(x_{n}+d_{n}\right)=A^{n+1}-\frac{1}{4} A^{n+1}-A^{n}-\frac{1}{4} A^{n}=A^{n}\left(\frac{3}{4} A-\frac{5}{4}\right)
$$

converges to $+\infty$ when $n \rightarrow+\infty$. Now applying Theorem 4.5 finishes the proof.

\section{ACKNOWLEDGEMENT}

I wish to thank B. Weiss for his valuable comments, which improved the final version of this paper.

\section{REFERENCES}

BP92 R. Benedetti and C. Petronio, Lectures on hyperbolic geometry (Springer, Berlin, 1992).

KLW04 D. Kleinbock, E. Lindenstrauss and B. Weiss, On fractal measures and diophantine approximation, Selecta Math. (NS) 10 (2004), 479-523.

KM98 D. Kleinbock and G. A. Margulis, Flows on homogeneous spaces and Diophantine approximation on manifolds, Ann. of Math. (2) 148 (1998), 339-360.

MU96 D. Mauldin and M. Urbański, Dimensions and measures in infinite iterated function systems, Proc. London Math. Soc. (3) 73 (1996), 105-154.

MU99 D. Mauldin and M. Urbański, Conformal iterated function systems with applications to the geometry of continued fractions, Trans. Amer. Math. Soc. 351 (1999), 4995-5025.

MU03a D. Mauldin and M. Urbański, Graph directed Markov systems: geometry and dynamics of limit sets (Cambridge University Press, 2003).

MU03b D. Mauldin and M. Urbański, The doubling property of conformal measures of infinite iterated function systems, J. Number Theory 102 (2003), 23-40.

PV A. Pollington and S. Velani, Metric Diophantine approximation and 'absolutely friendly' measure, Selecta Math. (NS), to appear.

PU F. Przytycki and M. Urbański, Fractals in the plane - ergodic theory methods (Cambridge University Press), to appear (available on author's webpage).

Urb01a M. Urbański, Porosity in conformal infinite iterated function systems, J. Number Theory $8 \mathbf{8}$ (2001), 283-312.

Urb01b M. Urbański, Rigidity of multi-dimensional conformal iterated function systems, Nonlinearity 14 (2001), 1593-1610.

Urb M. Urbański, Diophantine approximations and self-conformal measures, J. Number Theory, to appear. 


\section{DiOPHANTINE APPROXIMATION FOR CONFORMAL MEASURES}

Vee99 W. Veech, Measures supported on the set of uniquely ergodic directions of an holomorphic 1-form, Ergodic Theory Dynam. Systems 19 (1999), 1099-1109.

Wei01 B. Weiss, Almost no points on a Cantor set are very well approximable, R. Soc. Lond. Proc. Ser. A Math. Phys. Eng. Sci. 457 (2001), 949-952.

Mariusz Urbański urbanski@unt.edu, http://www.math.unt.edu/ urbanski

Department of Mathematics, University of North Texas, PO Box 311430, Denton, TX 76203-1430, USA 